\title{
Voltage Dependence of Precision Air Capacitors
}

\author{
J. Q. Shields
}

\author{
(August 1, 1965)
}

\begin{abstract}
Methods are presented for measuring both the relative and absolute voltage dependence of admittance standards. The practical circuits based on the methods are composed of precision bridges which are described in detail. Measurements with these circuits yield values for the proportional changes in admittance which result from specified changes in voltage. The results of measurements on selected capacitors are presented. Uncertainties are of the order of one part in $10^{9}$.

A general instability in the voltage dependence of precision air capacitors was observed at the higher accuracy levels. Most of the instability is believed to be caused by changes in the electrode surfaces.
\end{abstract}

Key Words: Bridge, capacitor, coaxial choke, difference transformer, electrode surfaces, thin films, three-terminal, transformer, voltage coefficient, voltage dependence, voltage ratio.

\section{Introduction}

Several years ago a computable cross capacitor, based on a theorem by A. M. Thompson and D. G. Lampard [1], ${ }^{1}$ was constructed at NBS [2]. The value of this capacitor is computable, in esu, within an estimated accuracy of 2 parts per million (ppm). A new cross capacitor of improved design is now being constructed. It is expected that this capacitor will be computable, in esu, within a few parts in $10^{8}$ or better.

The expected increase in accuracy not only requires better bridges and capacitors, but also requires that many sources of error be reevaluated. One such source of error is the voltage dependence of precision air capacitors.

To meet our particular needs, the quantity chosen as a measure of voltage dependence is termed $\gamma$ and is defined by the equation

$$
Y_{2}=Y_{1}(1+\gamma)
$$

where $Y_{1}$ is the admittance of a given standard at voltage $V_{1}, Y_{2}$ is the admittance of the same standard at voltage $V_{2}$, and the condition $V_{2}>V_{1}$ is imposed to restrict the meaning of $\gamma$. A given standard may have any number of values of $\gamma$, each with a specified voltage change. Since $\gamma$ represents a proportional change in admittance $(\Delta Y / Y)$, this measure of voltage dependence is in the preferred form for corrections to precision measurements.

In the past, most measurements of voltage dependence have been confined to a determination of the ratio, $\left(1+\gamma_{a}\right) /\left(1+\gamma_{b}\right)$, where $\gamma_{a}$ and $\gamma_{b}$ apply to different capacitors but have the same specified voltage change. One exception to the above is the work of N. L. Kusters and O. Petersons [3]. They developed

\footnotetext{
${ }^{1}$ Figures in brackets indicate the literature references at the end of this paper.
}

a method for measuring the voltage dependence of an individual capacitor, provided the voltage dependence results only from electrode deflections.

The accuracy requirements described earlier resulted in the need for a method which would be independent of the mechanisms which cause voltage dependence. Such a method is described in section 2 . The method yields values of $\gamma$ corresponding to a change in voltage from $V$ to $2 V$, where $V$ is a variable.

The practical circuits based on the method are described in sections 3 and 4 . These circuits were used to measure values of $\gamma$ for several capacitors corresponding to the following voltage changes: 12.5 to 25,25 to 50,50 to 100 , and 100 to $200 \mathrm{~V}$, rms, at $1592 \mathrm{~Hz}$. Measurement uncertainties had to be kept small since the above values of $\gamma$ are used to calculate other values of $\gamma$ in different voltage ranges such as from zero to $200 \mathrm{~V}$. The latter value is needed for the determination of the absolute unit of capacitance. Small uncertainties also provide the means for quickly detecting instabilities in the voltage dependence of capacitors. It was found that the voltage dependence of precision air capacitors was, in general, unstable at the accuracy level described. This subject is discussed in section 5 .

\section{Theory}

One method for measuring the voltage ratio of a transformer is to connect admittance standards to the transformer so as to form a bridge as shown in figure 1 [4]. In general, if the transformer has a nominally $m: n$ ratio, then $m+n$ nominally equal admittances are used, and $m+n$ detector balances are required corresponding to a cyclic permutation of these admittances. In this sequence of balances each admittance appears with voltage $E_{1} m$ times, and with voltage $E_{2} n$ times. If $E_{1} \neq E_{2}$, a ratio measurement error results since the admittance of a practical standard changes when the voltage applied to it 


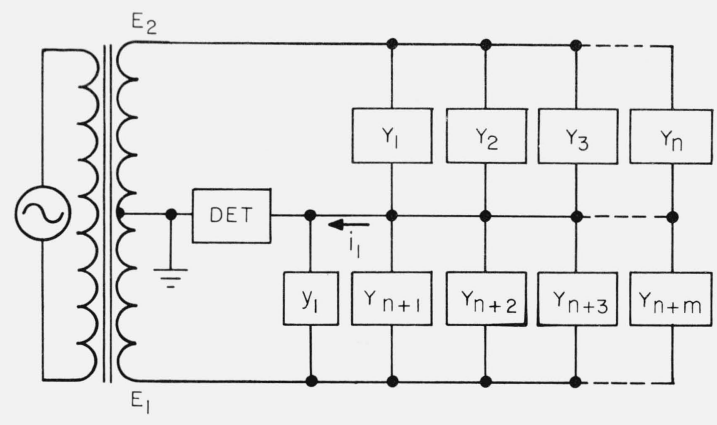

FIGURE 1. Circuit for measuring the voltage ratio of a transformer [4].

is changed. The difference between the measured ratio and the true ratio is related to the average change in admittance of the standards.

If the transformer ratio can be determined from a second set of measurements which is independent of voltage, the combination of the two sets of measurements will yield a value for the average change in admittance of the $m+n$ standards corresponding to a change in voltage from $E_{1}$ to $E_{2}$. The change in admittance of each standard can then be determined by measuring the relative changes between various pairs of standards.

The basis of this paper is a method of the type described above in which the second set of measurements consists of determining a group of $1: 1$ ratios which can be combined to determine the $m: n$ ratio of a transformer. For simplicity, a transformer having a $2: 1$ ratio was chosen so that only two $1: 1$ ratios need be determined.

It is assumed that taps are brought out from the secondary winding of a bridge transformer [5] so as to obtain three secondary voltages of approximately equal magnitude. These voltages are represented by $E_{A}, E_{B}$, and $E_{C}$ in figure 2 . The circuit shown in figure 2 consists of three bridges, each of which may be balanced, in turn, by the small, adjustable voltage $e$. For the case in which the circuit elements are closely matched, $e / E_{A} \equiv q \ll 1$, and only a low accuracy calibration of $q$ is needed for high accuracy determinations of ratios such as $E_{B} / E_{A}$.

It is assumed that the changes in $Y_{1}, Y_{2}, Y_{3} \ldots Y_{7}$ which result from changes in $e$ can be neglected. Following from this, we may consider the voltages applied to $Y_{5}, Y_{6}$, and $Y_{7}$ to be either $E_{A}$ or $2 E_{A}$ and define the values of $Y_{5}, Y_{6}$, and $Y_{7}$ to be $Y_{5}^{\prime}, Y_{6}^{\prime}$, and $Y_{7}^{\prime}$ at yoltage $E_{A}$, and $Y_{5}^{\prime}\left(1+\gamma_{5}\right), Y_{6}^{\prime}\left(1+\gamma_{6}\right)$, and $Y_{7}^{\prime}\left(1+\gamma_{7}\right)$ at voltage $2 E_{A}$.

The balance equations for bridge 1 are

$$
\begin{aligned}
& \frac{Y_{1}}{Y_{2}}=\frac{E_{A}-e_{1}}{E_{B}+e_{1}}=\frac{1-q_{1}}{1+\delta_{B}+q_{1}} \\
& \frac{Y_{2}}{Y_{1}}=\frac{E_{A}-e_{2}}{E_{B}+e_{2}}=\frac{1-q_{2}}{1+\delta_{B}+q_{2}}
\end{aligned}
$$

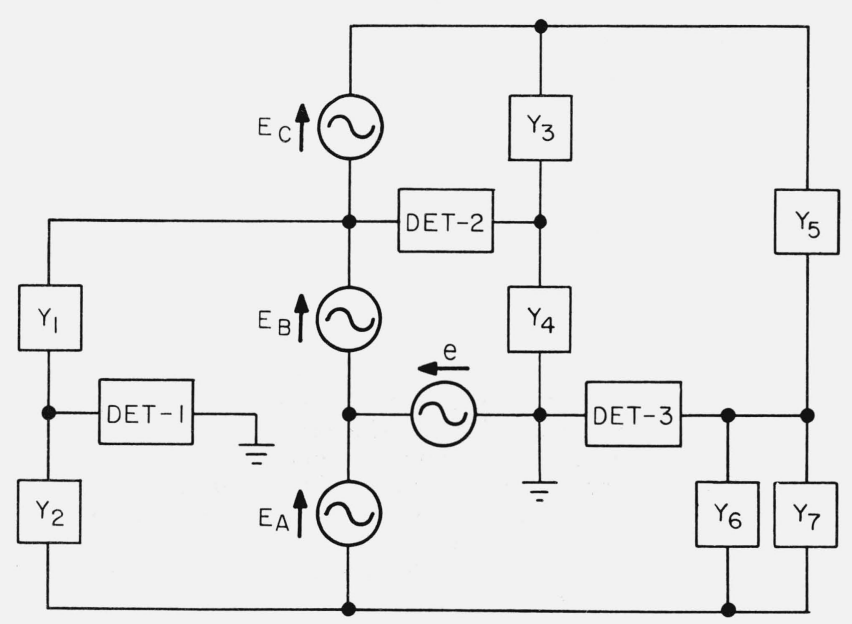

Figure 2. Basic three-bridge-circuit.

where $\frac{E_{B}}{E_{A}} \equiv 1+\delta_{B}$. Eliminating $Y_{1}$ and $Y_{2}$, we obtain

$$
\delta_{B}=-\left(q_{1}+q_{2}\right) .
$$

The balance equations for bridge 2 are

$$
\begin{aligned}
& \frac{Y_{3}}{Y_{4}}=\frac{E_{B}+e_{3}}{E_{C}}=\frac{1+\delta_{B}+q_{3}}{1+\delta_{C}} \\
& \frac{Y_{4}}{Y_{3}}=\frac{E_{B}+e_{4}}{E_{C}}=\frac{1+\delta_{B}+q_{4}}{1+\delta_{C}}
\end{aligned}
$$

where $\frac{E_{C}}{E_{A}} \equiv 1+\delta_{C}$. Eliminating $Y_{3}$ and $Y_{4}$, we obtain

$$
\delta_{C}=\frac{q_{3}+q_{4}+2 \delta_{B}}{2}+\epsilon_{1}
$$

where $\epsilon_{1}$ is a small correction term consisting of second and higher order terms in $q$.

The balance equations for bridge 3 are

$$
\begin{aligned}
& \frac{Y_{5}^{\prime}\left(1+\gamma_{5}\right)}{Y_{6}^{\prime}+Y_{7}^{\prime}}=\frac{E_{A}-e_{5}}{E_{B}+E_{C}+e_{5}}=\frac{1-q_{5}}{2+\delta_{B}+\delta_{C}+q_{5}} \\
& \frac{Y_{6}^{\prime}\left(1+\gamma_{6}\right)}{Y_{7}^{\prime}+Y_{5}^{\prime}}=\frac{E_{A}-e_{6}}{E_{B}+E_{C}+e_{6}}=\frac{1-q_{6}}{2+\delta_{B}+\delta_{C}+q_{6}} \\
& \frac{Y_{7}^{\prime}\left(1+\gamma_{7}\right)}{Y_{5}^{\prime}+Y_{6}^{\prime}}=\frac{E_{A}-e_{7}}{E_{B}+E_{C}+e_{7}}=\frac{1-q_{7}}{2+\delta_{B}+\delta_{C}+q_{7}} .
\end{aligned}
$$

Eliminating $Y_{5}^{\prime}, Y_{6}^{\prime}$, and $Y_{7}^{\prime}$, we obtain

$$
\bar{\gamma}=-\frac{\delta_{B}+\delta_{C}+q_{5}+q_{6}+q_{7}}{2}+\epsilon_{2} .
$$

where

$$
\bar{\gamma} \equiv \frac{\gamma_{5}+\gamma_{6}+\gamma_{7}}{3}
$$


and $\epsilon_{2}$ is a small correction term consisting of second and higher order terms in $q$ and $\gamma$.

Solving eqs (3), (6), and (10), we obtain

$\bar{\gamma}=\frac{4 q_{1}+4 q_{2}-q_{3}-q_{4}-2 q_{5}-2 q_{6}-2 q_{7}}{4}-\frac{\epsilon_{1}-2 \epsilon_{2}}{2}$.

In order to separate real and imaginary parts, let

$$
\gamma \equiv \gamma^{\prime}-j \gamma^{\prime \prime}
$$

and

$$
q \equiv q^{\prime}-j q^{\prime \prime}
$$

so that

$\overline{\gamma^{\prime}}=\frac{4 q_{1}^{\prime}+4 q_{2}^{\prime}-q_{3}^{\prime}-q_{4}^{\prime}-2 q_{5}^{\prime}-2 q_{6}^{\prime}-2 q_{7}^{\prime}}{4}-\frac{\epsilon_{1}^{\prime}-2 \epsilon_{2}^{\prime}}{2}$

and

$\bar{\gamma}^{\prime \prime}=\frac{4 q_{1}^{\prime \prime}+4 q_{2}^{\prime \prime}-q_{3}^{\prime \prime}-q_{4}^{\prime \prime}-2 q_{5}^{\prime \prime}-2 q_{6}^{\prime \prime}-2 q_{7}^{\prime \prime}}{4}-\frac{\epsilon_{1}^{\prime \prime}-2 \epsilon_{2}^{\prime \prime}}{2}$

For the capacitors and accuracies described in this paper, $\gamma^{\prime}$ is equal to the proportional change in capacitance $(\Delta C / C)$, and $\gamma^{\prime \prime}$ is equal to the change in dissipation factor, corresponding to a specified increase in voltage.

The values of $\gamma_{5}, \gamma_{6}$, and $\gamma_{7}$ can be separated from the value of $\bar{\gamma}$ by measuring the differences, $\gamma_{5}-\gamma_{6}$, $\gamma_{6}-\gamma_{7}$, and $\gamma_{7}-\gamma_{5}$. Measurements of this type are described in section 4. Combining the differences, we obtain

$$
\begin{aligned}
& \gamma_{5}=\bar{\gamma}+\left(\gamma_{5}-\gamma_{6}\right) / 3-\left(\gamma_{7}-\gamma_{5}\right) / 3 \\
& \gamma_{6}=\bar{\gamma}+\left(\gamma_{6}-\gamma_{7}\right) / 3-\left(\gamma_{5}-\gamma_{6}\right) / 3 \\
& \gamma_{7}=\bar{\gamma}+\left(\gamma_{7}-\gamma_{5}\right) / 3-\left(\gamma_{6}-\gamma_{7}\right) / 3 .
\end{aligned}
$$

\section{Practical Three-Bridge-Circuit}

The method described in the previous section was used to determine the voltage dependence of three 100 picofarad $(\mathrm{pF})$ air capacitors, designated 100A, 100B, and 100C. This section describes the equipment and techniques used to determine the values of

$$
\bar{\gamma}_{100} \equiv \frac{\gamma_{100 \mathrm{~A}}+\gamma_{100 \mathrm{~B}}+\gamma_{100 \mathrm{C}}}{3}
$$

corresponding to the following voltage changes: 12.5 to 25,25 to 50,50 to 100 , and 100 to $200 \mathrm{~V}$, rms, at $1592 \mathrm{~Hz}$.

If small capacitors are to be well defined, they must be shielded. The addition of shielding to the circuit of figure 2 is shown in figures $3 \mathrm{a}, 3 \mathrm{~b}$, and $3 \mathrm{c}$. Each of the admittance standards represented in figures $3 a$, $3 \mathrm{~b}$, and $3 \mathrm{c}$ consists of a completely shielded threeterminal air capacitor connected to a pair of coaxial

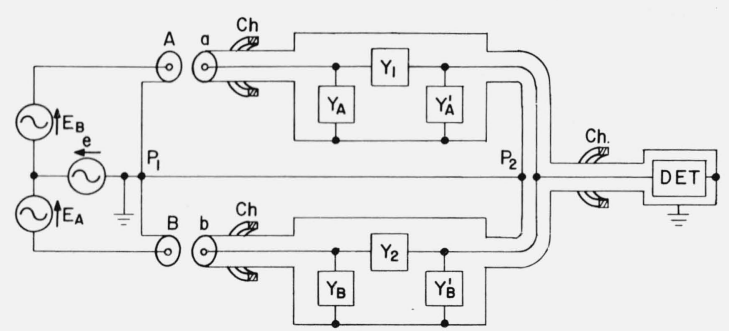

FIGURE 3a. Bridge 1 of the practical three-bridge-circuit.

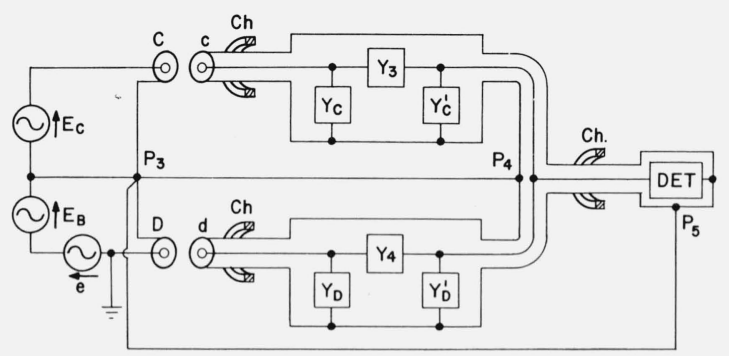

FigURE 3b. Bridge 2 of the practical three-bridge-circuit.

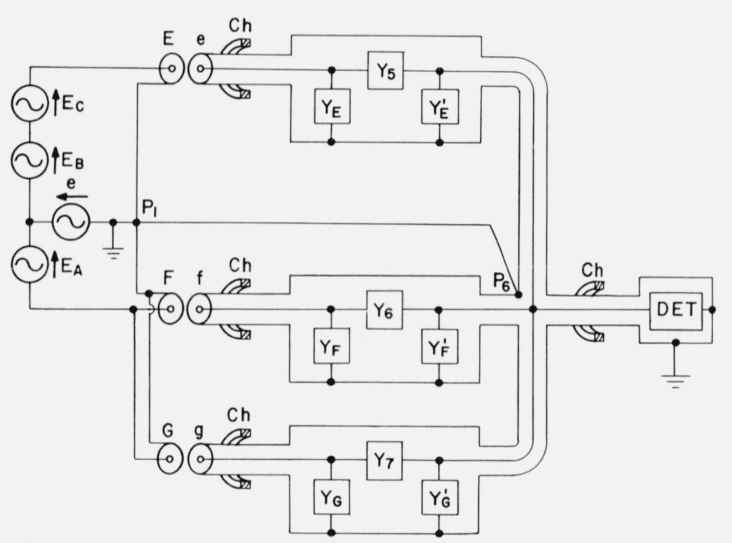

FIGURE 3c. Bridge 3 of the practical three-bridge-circuit.

cables. The defining terminals of each standard are located at one of the detector junctions and at one of the coaxial connectors designated $a, b, c \ldots g$.

The required circuits are obtained by joining connectors $a, b, c \ldots g$ to connectors, $A, B, C \ldots G$ in various configurations. When connector $a$ is joined to connector $A$ and $b$ to $B$, the balance equation is given by equation (1), section 2 . When $a$ is joined to $B$ and $b$ to $A$, the balance equation is given by eq (2). Similarly, eqs (4) and (5) are applicable to figure $3 \mathrm{~b}$ and eqs (7), (8), and (9) are applicable to figure 3c. For purposes of evaluating the effects of self and mutual impedances in the leads (not shown in the figures), it will initially be assumed that: (1) net cur- 
rents through the "coaxial chokes" [6], designated Ch in the figures, are negligibly small, (2) the voltages at connector $A$ are, to sufficient accuracy, independent of whether $A$ is joined to $a$ or to $b$, and similarly for the voltages at connectors $B, C, D \ldots G,(3)$ only negligible effects result from externally induced emfs and external loading, e.g., capacitance from the bridge shielding to its surroundings.

Subject to the above assumptions, the two values of $e$ corresponding to the two balance conditions for bridge 1 yield a measure of the voltage ratio, $V_{A} / V_{B}$ where $V_{A}$ is the voltage between the inner and outer terminals of connector $A$ with $e=0$ and $V_{B}$ is the voltage between the inner and outer terminals of connector $B$ with $e=0$. Similarly, a measure of the voltage ratios, $V_{C} / V_{D}$ and $V_{E} / V_{F}$ are obtained using bridges 2 and 3 , respectively. The value of $\bar{\gamma}_{100}$ is calculated from the measured values of $V_{A} / V_{B}, V_{C} / V_{D}$, and $V_{E} / V_{F}$. It is assumed that the relationship between voltages $V_{A}, V_{B}, V_{C} \ldots V_{G}$ is known. Ideally, the voltage drops in the leads which precede connectors $A, B$, $C$. . G $G$ should be zero, but if the voltage drops are small, eq (12) is sufficiently accurate when the relationship between the voltages is as follows: $V_{A}=-V_{D}$, $V_{B}=V_{F}=V_{G}, \quad V_{E}=V_{A}+V_{C}$. Since voltage ratios rather than voltages are measured, other satisfactory relationships between the voltages can be described, e.g., $V_{C}$ and $V_{D}$ can each be changed if $V_{C} / V_{D}$ remains unchanged. Thus, the error in the measurement of $\bar{\gamma}_{100}$ which results from voltage drops in the leads will be small if the impedances in the leads which join various terminals of connectors $A, B, C \ldots G$ are small or properly matched. These features are present in the junction box shown within broken lines in figure 4. Parallel lines represent copper strips separated by thin strips of insulation.

The error in the measurement of $\bar{\gamma}_{100}$ resulting from lead impedances within the junction box and resulting from contact impedances between connectors $A, B$, $C \ldots G$ and connectors $a, b, c \ldots g$ was found to be approximately $1 \times 10^{-9}$. Most of this error was cor-

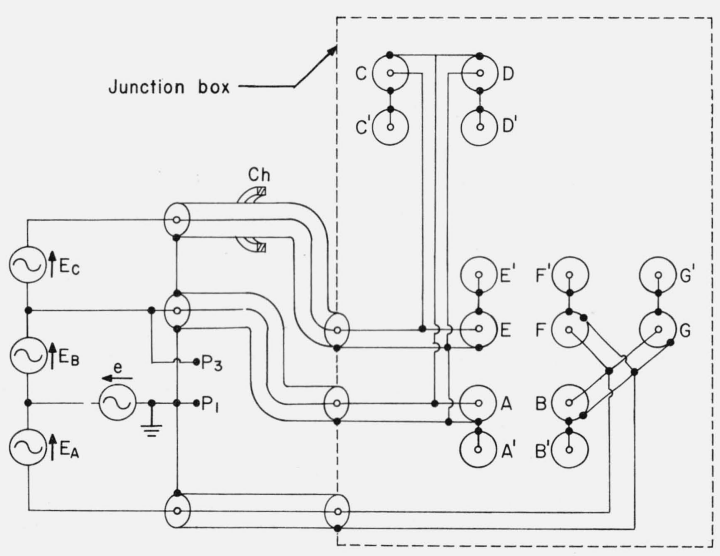

FiguRE 4. Source of bridge voltages.

Junction box (within broken lines) connected by codxial cables to an "active direct. reading ratio set" [7]. rected for by changing the currents at the terminals of connectors $a, b, c \ldots g$ and observing the change in the measured value of $\bar{\gamma}_{100}$. Since the current at the inner or outer terminal of connector $a$ was nearly proportional to the internal load, $Y_{1}+Y_{A}$, and similarly for the currents at the terminals of $b, c, d, \ldots g$, the following procedure was used: (1) $Y_{A}, Y_{B}, Y_{C} \ldots Y_{G}$ were adjusted so that each of the seven internal loads, $Y_{1}+Y_{A}, Y_{2}+Y_{B}, Y_{3}+Y_{C} \ldots Y_{7}+Y_{G}$, were increased by a known factor, (2) the change in the measured value of $\bar{\gamma}_{100}$ was used to calculate a third value of $\bar{\gamma}_{100}$ corresponding to the internal loads being zero. The above procedure amounts to an extrapolation of the effect of internal loads to zero.

The internal loads were measured by disconnecting $A, B, C \ldots G$ from $a, b, c \ldots g$, shorting the detectors, and measuring the two-terminal admittances between the inner and outer terminals of connectors $a$, $b, c \ldots g$. Since the lead impedances within the junction box were small, low accuracy measurements were sufficient for purposes of extrapolating the internal loads to zero. The larger equivalent lead impedances in that portion of the circuit which preceded the junction box (see fig. 4) required that more accurate measurements be made for purposes of adjusting $Y_{B}, Y_{D}, Y_{F}$, and $Y_{G}$ so that $Y_{1}+Y_{A}=Y_{2}+Y_{B}$, $Y_{3}+Y_{C}=Y_{4}+Y_{D}$, and $Y_{5}+Y_{E}=Y_{6}+Y_{F}=Y_{7}+Y_{G}$. The accuracy of adjustment needed was determined by connecting additional loads between the inner and outer terminals of $A, B, C \ldots G$, each in turn, and noting the changes in $e$ required to rebalance the bridges. It was found that if the internal loads were adjusted within $\pm 1 \times 10^{-9}$ mho, the voltages at connector $A$ were, to sufficient accuracy, independent of whether $A$ was joined to $a$ or to $b$, and similarly for the voltages at $B, C, D \ldots G$.

The coaxial chokes used consisted of a number of turns of the coaxial cable threaded through a high permeability core. The resulting impedance to net current in the cable was about $400 \Omega$ at $1592 \mathrm{~Hz}$. Since the impedance in the path, $P_{1}-A-a-P_{2}$, of figure $3 \mathrm{a}$ was about $0.1 \Omega$, net current through the coaxial choke was of the order of a few parts in $10^{4}$ of the current in the inner or outer conductor of the cable [6]. The impedances in the paths, $P_{1}-A-a$ and $P_{1}-B-b$, differed by less than $2 \mathrm{~m} \Omega$. The maximum internal load was about $3000 \mathrm{pF}$.

Thus far in the discussion the effects of externally induced emfs and external loading have been neglected. Although these effects were small in the practical circuit used, they were not negligible. Appropriate corrections were determined from additional measurements which consisted of recording the unbalance at the detectors when the admittance standards of each bridge, in turn, were joined to the shorting connectors, $A^{\prime}, B^{\prime}, C^{\prime}, \ldots . G^{\prime}$, (see fig. 4) while the other standards were joined to $A, B, C, \ldots G$. Since the accuracy of the corrections depended on the size of the unbalance at each detector, it was desirable for the effects of externally induced emfs and external loads to be small. The techniques used to reduce these effects will be considered next. 


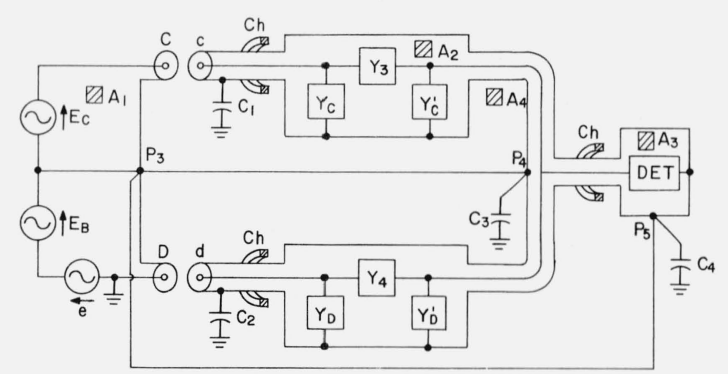

FigURE 5. Bridge 2 redrawn to illustrate external loads and sources of induced emf.

Bridge 2 has been redrawn in figure 5 for purposes of illustrating the different types of induced emf's and external loads. Since the shielding of bridge 2 is not at ground potential, the largest external loads are associated with this bridge. External loads are represented by $C_{1}, C_{2}, C_{3}$, and $C_{4}$ in figure 5 . Induced emfs are represented indirectly by showing areas in which a time-varying magnetic flux is assumed to exist. These areas are designated $A_{1}, A_{2}, A_{3}$, and $A_{4}$ in figure 5.

Most of the flux through $A_{1}$ existed in the region preceding the junction box and thus had no effect on the measured value of $\bar{\gamma}_{100}$. Flux through $A_{2}$ was reduced by using coaxial cables of sufficient length so that the three-terminal capacitor could be positioned at a distance from sources of appreciable magnetic field. Flux through $A_{3}$ was reduced by using coaxial cables and magnetic shielding in that portion of the detector circuit which precedes voltage amplification. The effects of flux through $A_{4}$ were reduced by the use of coaxial chokes, e.g., if the high permeability core positioned between connector $c$ and $P_{4}$ were removed from the circuit, the current through $Y_{3}$ which results from flux through $A_{4}$ would be increased by a factor of a few thousand.

The effects of external loads were reduced by the use of coaxial chokes in conjunction with low-impedance leads which bypass the junction box (hereafter termed bypass leads). The lead connecting $P_{3}$ to $P_{4}$ and the lead connecting $P_{3}$ to $P_{5}$ are examples of bypass leads in figure 5 . These leads, which have impedances of about $0.1 \Omega$, supply most of the current to $C_{3}$ and $C_{4}$. Thus, if coaxial chokes and bypass leads are properly positioned, voltage drops will be reduced in critical portions of the circuit such as the junction box leads and the cables of the admittance standards and detector circuit.

The effects of external loads were also reduced by the use of an electrostatic shield which is represented by a broken line in figure 6 . This figure represents more closely the actual circuit used. The electrostatic shield, maintained at a potential of approximately $E_{B}$ with respect to ground, encloses those circuit elements which would otherwise have a large capacitance to the grounded surroundings. Current to the shield is supplied by the bypass lead connected to $P_{3}$.

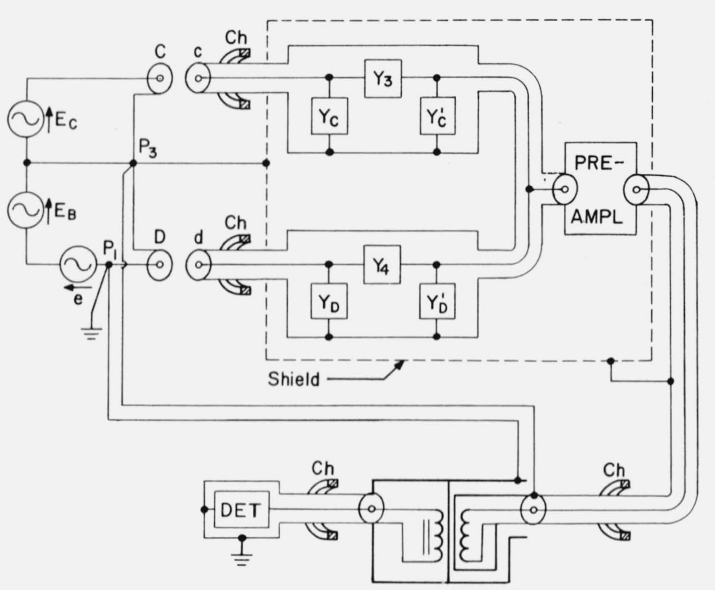

FIGURE 6. Bridge 2 redrawn to show the difference transformer and additional shielding.

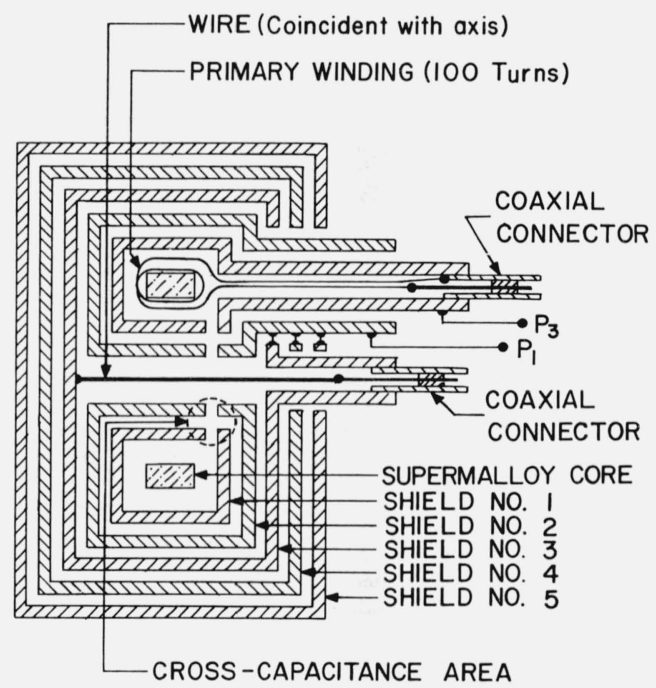

FIgURE 7. Cross section through the axis of the difference transformer.

The transformer shown in figure 6 is termed a difference transformer ${ }^{2}$ and is used to change the potential of the detector cables with respect to ground from approximately $\boldsymbol{E}_{B}$ to zero. This was necessary in order that a phase-sensitive detector having a grounded power supply could be used. An adequate signal-tonoise ratio was maintained by using a battery-operated preamplifier having a voltage amplification of approximately $10^{3}$ (see fig. 6). Coaxial chokes assured that the bypass leads in figure 6 supplied most of the current to the large capacitance which existed between the shielding of the difference transformer.

A cross-sectional view of the difference transformer is shown in figure 7 . The secondary winding is a

${ }^{2}$ The basic ideas in the transformer are similar to those in a transformer built by A. M. Thompson and termed by him a difference transformer. 
single turn consisting of shield No. 3 connected to a wire through the center of the core. With the exception of shield No. 4 which is mu-metal, all shielding is brass.

Most of the current resulting from the capacitance between shield No. 1 and shield No. 2 does not encircle the Supermalloy core. The current which does encircle the core, if not compensated, would induce a voltage in the detector winding. The two capacitances which result in core excitation are associated with the corners of the shield gaps. With reference to the cross-capacitance area shown in figure 7 , the two capacitances are (1) from the upper left to the lower right, (2) from the upper right to the lower left. It can be seen that the two currents encircle the core in opposite senses, and hence their effects can be made to cancel by proper adjustment of the gap geometry. The gap was adjusted experimentally by observing the detector voltage with the transformer input open circuited and with the bypass leads connected.

As a result of the techniques described above, the unbalance at the detectors was small when the admittance standards of each bridge, in turn, were joined to the shorting connectors, $A^{\prime}, B^{\prime}, C^{\prime}$. . . $G^{\prime}$, while the other standards were joined to $A, B, C$ ...G. Thus, accurate corrections were obtained for most of the effects of induced emf's and external loads. Those effects which were not accounted for in the corrections will be considered next.

Since currents were reduced when the standards were connected to the shorting terminals $A^{\prime}, B^{\prime}$, $C^{\prime}, \ldots G^{\prime}$, induced emf's resulting from currents in that portion of the circuit shown in figure 4 were not entirely accounted for in the above corrections. The use of coaxial cables, parallel-strip leads, and magnetic shielding assured that the resulting errors were negligible. It is interesting to note that if the errors had not been negligible, they would have been accounted for in the extrapolation of the internal loads to zero. The effect of induced emf's resulting from currents within the standards was negligible since the phase of the induced emf's was shifted by $180^{\circ}$ when the standards were permuted.

Magnetic fields in the vicinity of the junction box may induce emf's which are selective with respect to connectors $A, B, C \ldots G$, thus causing errors in the measurement of $\bar{\gamma}_{100}$. Induced emf's of this type were kept small by using closely spaced copper strips in the construction of the junction box (see fig. 4). The following procedure was used to assure that the remaining errors were negligible: (1) A small probe was used to measure the magnetic field in the vicinity of the junction box, including the region where changes in geometry occur due to changes in the connections of the standards to the junction box, (2) the current in a loop of wire was adjusted so as to increase the measured field by a factor of approximately $100,(3)$ the changes in $e$ required to rebalance the bridges were related to changes in $\bar{\gamma}_{100}$.
The external loads, $C_{1}$ and $C_{2}$ in figure 5 , represent the capacitances between the grounded surroundings and those cables which are located between the coaxial chokes and connectors $c$ and $d$. The circuit was designed so that $C_{1}$ and $C_{2}$ would be small and nearly equal. Measurements of $C_{1}$ and $C_{2}$ and of the lead impedances in the junction box indicated that the resulting error in the measurement of $\bar{\gamma}_{100}$ was small. This error is accounted for in item 1 , table 1 . Also accounted for in item 1 , table 1 are errors resulting from capacitance between the copper strips of the junction box and errors resulting from imperfect balancing of the internal loads.

TABLE 1. Uncertainties in the measurement of $\bar{\gamma}_{100} \equiv \bar{\gamma}_{100}^{\prime}-\mathrm{j} \bar{\gamma}_{100}^{\prime \prime}$

\begin{tabular}{|c|c|c|}
\hline Sources of error & $\begin{array}{c}\text { Systematic errors in } \\
\bar{\gamma}_{100}^{\prime}\end{array}$ & $\begin{array}{c}\text { Systematic errors in } \\
\bar{\gamma}_{100}^{\prime \prime}\end{array}$ \\
\hline 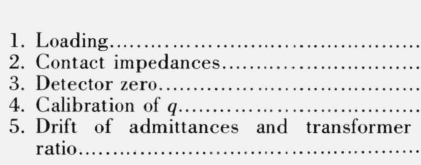 & $\begin{array}{c}\text { Parts in } 10^{10} \\
1 \\
\quad \cdots \ldots . . . \\
1 \\
2 \\
2\end{array}$ & $\begin{array}{c}\text { Parts in } 10^{10} \\
1 \\
2 \\
1 \\
1 \\
1\end{array}$ \\
\hline 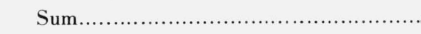 & 6 & 6 \\
\hline
\end{tabular}

Standard deviation of $\bar{\gamma}_{100}^{\prime}$ or $\bar{\gamma}_{100}^{\prime \prime}$ is $3 \times 10^{-10}$

Table 1 lists only the significant errors which were not corrected for by extrapolating the internal loads to zero or by using the shorting terminals, $A^{\prime}, B^{\prime}$, $C^{\prime} \ldots G^{\prime}$, as described earlier. Item 2 , table 1 , accounts for small changes in the contact impedances between $a, b, c \ldots g$ and $A, B, C, \ldots G$ which were found to occur with continued use. Item 3 , table 1 , accounts for possible systematic errors which result from observer bias in determining the null balance condition.

Item 4 , table 1 , accounts for errors in the calibration of the instrument used to obtain $E_{A}, E_{B}, E_{C}$, and $e$. The essential features of the instrument, termed an "active direct-reading ratio set," are described in reference [7]. The adjustments of $q \equiv \frac{e}{E_{A}} \equiv q^{\prime}-j q^{\prime \prime}$ were made by adjusting two sets of dials, one corresponding to changes in $q^{\prime}$ and the other to changes in $q^{\prime \prime}$. The maximum range of $q^{\prime}$ or $q^{\prime \prime}$ was $\pm 10 \mathrm{ppm}$ with a minimum adjustment of $2 \times 10^{-10}$.

Item 5 , table 1 , accounts for errors which result from the admittances and transformer ratio having a nonconstant drift rate and from observer bias in timing the bridge balances which were made so as to obtain values of $q$ in the following sequence: $q_{3}, q_{4}, q_{3} ; q_{1}, q_{2}$, $q_{1} ; q_{5}, q_{6}, q_{7}, q_{6}, q_{5} ; q_{1}, q_{2}, q_{1} ; q_{3}, q_{4}, q_{3}$. The above set of values could be obtained in about 12 minutes with $E_{A}=100 \mathrm{~V}$, rms. The standard deviations of $\bar{\gamma}_{100}^{\prime}$ and $\bar{\gamma}_{100}^{\prime \prime}$, obtained from one such set, were about $6 \times 10^{-10}$. Slightly larger standard deviations were obtained at lower voltages. A sufficient number of measurements was made so that the final values of $\bar{\gamma}_{100}^{\prime}$ and $\bar{\gamma}_{100}^{\prime \prime}$ had standard deviations of about $3 \times 10^{-10}$. 


\section{Difference Measurements}

The difference in voltage dependence between two standards whose admittances are approximately equal can be determined from ordinary bridge measurements, e.g., simple substitution combined with a change in bridge voltage. The particular circuit used for this type of difference measurement is described in section 4.1.

When the admittances of the two standards are radically different, additional measurements are required. These measurements are described in section 4.2 .

\subsection{Difference Measurements Involving Approximately Equal Admittances}

The circuit shown in figure $3 \mathrm{a}$ is typical of the circuits used for difference measurements. The two general balance equations for this bridge have already been presented, namely eqs (1) and (2). For the case in which the voltage, $E_{A}$, has two specific values, $E_{A L}$ and $E_{A H}$, four specific balance equations may be written:

$$
\begin{aligned}
& \frac{Y_{1 L}}{Y_{2 L}}=\frac{E_{A L}-e_{1 L}}{E_{B L}+e_{1 L}}=\frac{1-q_{1 L}}{1+\delta_{B L}+q_{1 L}} \\
& \frac{Y_{2 L}}{Y_{1 L}}=\frac{E_{A L}-e_{2 L}}{E_{B L}+e_{2 L}}=\frac{1-q_{2 L}}{1+\delta_{B L}+q_{2 L}} \\
& \frac{Y_{1 H}}{Y_{2 H}}=\frac{E_{A H}-e_{1 H}}{E_{B H}+e_{1 H}}=\frac{1-q_{1 H}}{1+\delta_{B H}+q_{1 H}} \\
& \frac{Y_{2 H}}{Y_{1 H}}=\frac{E_{A H}-e_{2 H}}{E_{B H}+e_{2 H}}=\frac{1-q_{2 H}}{1+\delta_{B H}+q_{2 H}} .
\end{aligned}
$$

If

$$
Y_{1 H} \equiv Y_{1 L}\left(1+\gamma_{1}\right)
$$

and

$$
Y_{2 H} \equiv Y_{2 L}\left(1+\gamma_{2}\right)
$$

then

$$
\gamma_{1}-\gamma_{2}=\left(q_{2 H}-q_{2 L}\right)-\left(q_{1 H}-q_{1 L}\right)+\epsilon_{3}
$$

where $\epsilon_{3}$ is a small correction term consisting of second and higher order terms in $q$ and $\gamma$.

The method described above was used to measure each of the following differences: $\gamma_{100_{\mathrm{A}}}-\gamma_{100 \mathrm{~B}}, \gamma_{100_{\mathrm{B}}}$ $-\gamma_{100 \mathrm{C}}, \quad \gamma_{100 \mathrm{C}}-\gamma_{100_{\mathrm{A}}}, \quad \gamma_{1000_{\mathrm{A}}}-\gamma_{1000 \mathrm{~B}}, \quad \gamma_{1000 \mathrm{~B}}-\gamma_{1000 \mathrm{C}}$, and $\gamma_{1000 \mathrm{c}}-\gamma_{1000 \mathrm{~A}}$, where the subscripts refer to three $100 \mathrm{pF}$ air capacitors and three $1000 \mathrm{pF}$ air capacitors designated $100 \mathrm{~A}, 100 \mathrm{~B}, 100 \mathrm{C}$, and $1000 \mathrm{~A}, 1000 \mathrm{~B}$, $1000 \mathrm{C}$, respectively. Four values were obtained for each of the above differences corresponding to the following voltage changes: 12.5 to 25,25 to 50,50 to 100 , and 100 to $200 \mathrm{~V}$, rms, at $1592 \mathrm{~Hz}$. The discussion of measurement uncertainties which follows is applicable to each of these values.
Since the differences, $q_{2 H}-q_{2 L}$ and $q_{1 H}-q_{1 L}$, were small and of opposite sign in eq (27), most errors of the type described in section 3 were negligible. The only exceptions were errors resulting from drift of the admittances and transformer ratio. These errors were partially reduced by the measuring sequence which follows: $q_{1 L}, q_{1 H}, q_{1 L}, q_{2 L}, q_{2 H}, q_{2 L}$. Rather than attempting to obtain a precise bridge balance at a specified time, each balance in the above sequence was made as quickly as possible. Nonuniform timing was almost inevitable since the detector sensitivity was better at high voltage. The resulting errors were kept small by injecting a small current to compensate the effect of capacitor drift. This was accomplished with an auxiliary circuit, termed a "drifter circuit."

If no adjustments of $e$ are made, the relative changes in capacitance with time result in a time-variation in the amplitude of the current at the detector. The drifter circuit is used to inject a compensating current at the detector. The basic part of the circuit is a threeterminal, variable, air capacitor having a maximum capacitance of $10 \mathrm{pF}$. The adjusting shaft of the capacitor is connected to a constant-speed motor with speed reducer, thus providing a capacitance which varies linearly with time. Coaxial cables are used to connect the terminals of the capacitor to the detector junction and to an inductive voltage divider whose voltage is supplied by the "active direct-reading ratio-set" used to obtain $E_{A}, E_{B}$, and $e$.

The amplitude of the current injected at the detector has a first derivative with respect to time which can be adjusted by adjusting the inductive voltage divider and a second derivative which is zero. Since the drift rate of the admittance standards changed only slightly during the time needed for a set of detector balances, the use of the drifter circuit resulted in appreciable reductions in the time rate of change of $e$ required to maintain a detector balance. In addition, the deviations from constant drift rate, which can result in systematic errors, were easily measured.

A sufficient number of measurements was made so that the standard deviation of each of the final values, $\gamma_{100_{\mathrm{A}}}^{\prime}-\gamma_{100 \mathrm{~B}}^{\prime}, \quad \gamma_{100_{\mathrm{A}}}^{\prime \prime}-\gamma_{100 \mathrm{~B}}^{\prime \prime}, \quad \gamma_{1000_{\mathrm{A}}}^{\prime}-\gamma_{1000_{\mathrm{B}}}^{\prime}$, etc., was $3 \times 10^{-10}$ or less. A systematic error of $2 \times 10^{-10}$ was assigned to each of the final values to account for incomplete compensation of the admittance and transformer ratio drift.

\subsection{Difference Measurements Involving Radically Different Admittances}

The circuit shown in frgure 8 is used to measure the difference,

$$
\bar{\gamma}_{a}-\bar{\gamma}_{b} \equiv \frac{\gamma_{8}+\gamma_{9}+\gamma_{10}}{3}-\frac{\gamma_{5}+\gamma_{6}+\gamma_{7}}{3}
$$

If connectors $Q, R$, and $S$ are permuted cyclically with respect to connectors $E, F$, and $G$ of the junction box (fig. 4), six different bridge balances can be made. 


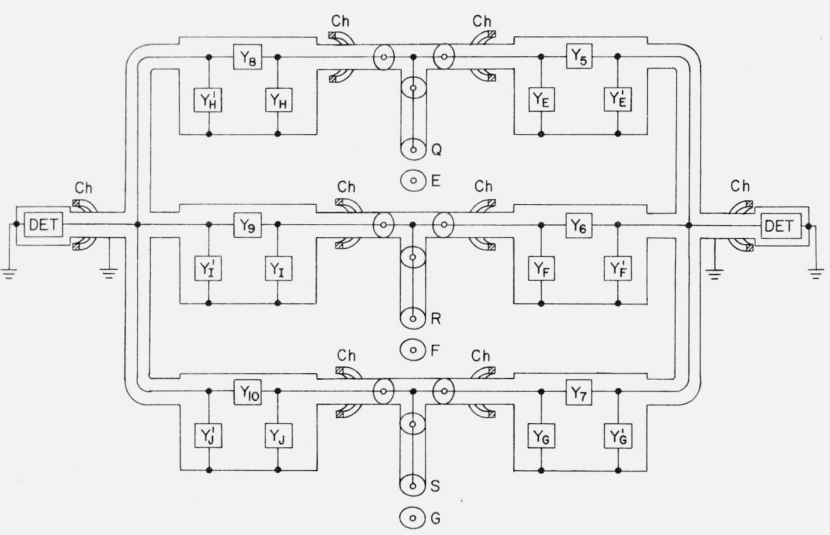

FIGURE 8. Circuit used for difference measurements with $\mathrm{Y}_{5} \approx \mathrm{Y}_{6} \approx \mathrm{Y}_{7} \neq \mathrm{Y}_{8} \approx \mathrm{Y}_{9} \approx \mathrm{Y}_{10}$.

Three of the six balance equations have already been presented, eqs (7), (8), and (9). The other three equations are easily obtained by changing the subscripts of $Y^{\prime}, \gamma, e$, and $q$ in eqs (7), (8), and (9). Solving the six equations, we obtain

$$
\bar{\gamma}_{a}-\bar{\gamma}_{b}=\frac{q_{5}+q_{6}+q_{7}-q_{8}-q_{9}-q_{10}}{2}+\epsilon_{4}
$$

where $\epsilon_{4}$ is a small correction term consisting of second and higher order terms in $\gamma$ and $q$.

The method described above was used to measure the difference,

$$
\begin{aligned}
& \bar{\gamma}_{1000}-\bar{\gamma}_{100} \equiv \frac{\gamma_{1000 \mathrm{~A}}+\gamma_{1000 \mathrm{~B}}+\gamma_{1000 \mathrm{C}}}{3} \\
&-\frac{\gamma_{100 \mathrm{~A}}+\gamma_{100 \mathrm{~B}}+\gamma_{100 \mathrm{C}}}{3}
\end{aligned}
$$

Four different values were obtained for the above difference, corresponding to the following changes in voltage: 12.5 to 25,25 to 50,50 to 100 , and 100 to $200 \mathrm{~V}$, rms, at $1592 \mathrm{~Hz}$. The discussion of measurement uncertainties which follows is applicable to each of these values.

Corrections were obtained for the effects of induced emf's and external loads (see section 3) by joining connectors $Q, R$, and $S$ to the shorting connectors, $E^{\prime}, F^{\prime}$, and $G^{\prime}$ (fig. 4). It was not necessary for the internal loads to be equal or to extrapolate the internal loads to zero. Errors resulting from admittance and transformer ratio drift were reduced by obtaining values of $q$ in the following sequence: $q_{5}, q_{6}, q_{7}, q_{6}$, $q_{5}, q_{8}, q_{9}, q_{10}, q_{9}, q_{8}, q_{5}, q_{6}, q_{7}, q_{6}, q_{5}$. A sufficient number of measurements was made so that the standard deviation of each of the differences, $\bar{\gamma}_{1000}^{\prime}-\bar{\gamma}_{100}^{\prime}$ and $\bar{\gamma}_{1000}^{\prime \prime}-\bar{\gamma}_{100}^{\prime \prime}$, was reduced to about $5 \times 10^{-10}$. In addition, an allowance of $5 \times 10^{-10}$ was assigned to each of the differences to account for systematic errors which may be described as similar to items 3,4 , and 5 in table 1.

\section{Results}

The values in tables 2,3 , and 4 were calculated from the results of measurements described in sections 3 and 4 . Capacitors $100 \mathrm{~A}, 100 \mathrm{~B}$, and $100 \mathrm{C}$ are of cylindrical construction, whereas capacitors $1000 \mathrm{~A}$, $1000 \mathrm{~B}$, and $1000 \mathrm{C}$ are of parallel-plate construction. Most of the voltage dependence of capacitors 1000A, $1000 \mathrm{~B}$, and $1000 \mathrm{C}$ is believed to be caused by electrode deflections [3]. Most of the voltage dependence of capacitors $100 \mathrm{~A}, 100 \mathrm{~B}$, and $100 \mathrm{C}$ is believed to be associated with the electrode surfaces.

The values in table 4 were obtained either immediately after assembly of the capacitors, one month after assembly, or eight months after assembly. The values in table 2 were obtained eight months after assembly. A comparison indicates that the values of $\gamma_{100 \mathrm{~A}}, \gamma_{100 \mathrm{~B}}$, and $\gamma_{100 \mathrm{C}}$ were probably smallest immediately after assembly. The changes in $\gamma$ are believed to have been caused by structural changes on the surfaces of the brass electrodes or by the migration of thin films onto the electrode surfaces from regions of the capacitor which were difficult to clean. A consideration of typical dimensions leads to the conclusion that sizeable effects could result from thin films, e.g., the ratio of molecular diameters to typical electrode separations is considerably larger than the desired accuracy of a few parts in $10^{9}$.

Measurements, similar to those in table 4 and with comparable accuracies, were also made on capacitors $1000 \mathrm{~A}, 1000 \mathrm{~B}$, and $1000 \mathrm{C}$. The largest change during the eight month period was $4 \times 10^{-10}$. Capacitors $1000 \mathrm{~A}, 1000 \mathrm{~B}$, and $1000 \mathrm{C}$ had been in use for a number of years prior to the measurements described. The electrodes were gold plated. These capacitors and capacitors $100 \mathrm{~A}, 100 \mathrm{~B}$, and $100 \mathrm{C}$ were evacuated with an oil sealed mechanical pump trapped with dry ice.

TABLE 2. Voltage dependence of capacitors $100 \mathrm{~A}, 100 \mathrm{~B}$, and $100 \mathrm{C}$

Values of $\gamma^{\prime}$ represent proportional changes in capacitance $(\Delta C / C)$. Values of $\gamma^{\prime \prime}$ represent

\begin{tabular}{|c|c|c|c|}
\hline Change in voltage & $\gamma_{19 \mathrm{O}_{\mathrm{A}}}^{\prime}$ & $\gamma_{10 \mathrm{OB}}^{\prime}$ & $\gamma_{100 \mathrm{C}}^{\prime}$ \\
\hline 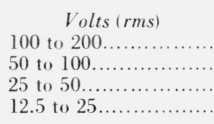 & $\begin{array}{c}\text { Parts in } 10^{9} \\
+1.9 \\
+.9 \\
+.3 \\
.0\end{array}$ & $\begin{array}{c}\text { Parts in } 10^{9} \\
\quad+8.0 \\
+2.9 \\
\quad+1.4 \\
+0.6\end{array}$ & $\begin{array}{c}\text { Parts in } 10^{9} \\
\quad+0.9 \\
+.6 \\
\quad+.4 \\
\quad+.1\end{array}$ \\
\hline Change in voltage & $\gamma_{100_{A}}^{\prime \prime}$ & $\gamma_{100_{B}}^{\prime \prime}$ & $\gamma_{100 \mathrm{c}}^{\prime \prime}$ \\
\hline 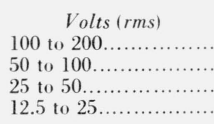 & $\begin{array}{c}\text { Parts in } 10^{9} \\
+0.1 \\
.0 \\
-.4 \\
.0\end{array}$ & $\begin{array}{c}\text { Parts in } 10^{9} \\
-0.4 \\
+.2 \\
-.5 \\
-.2\end{array}$ & $\begin{array}{c}\text { Parts in } 10^{9} \\
0.0 \\
+.1 \\
-.2 \\
-.3\end{array}$ \\
\hline
\end{tabular}
changes in dissipation factor. Standard deviation $=3 \times 10^{-10}$. Systematic error $<7 \times 10^{-10}$. 
TABLE 3. Voltage dependence of capacitors $1000 \mathrm{~A}, 1000 \mathrm{~B}$, and $1000 \mathrm{C}$

\begin{tabular}{|c|c|c|c|}
\hline Change in voltage & $\gamma_{1000 \mathrm{~A}}^{\prime}$ & $\gamma_{1000 \mathrm{~B}}^{\prime}$ & $\gamma_{1000 \mathrm{C}}^{\prime}$ \\
\hline $\begin{array}{c}\quad \text { Volts (rms) } \\
100 \text { to } 200 \ldots \ldots \ldots \ldots \ldots \\
50 \text { to } 100 \ldots \ldots \ldots \ldots \ldots \ldots \ldots \ldots \ldots \\
25 \text { to } 50 \ldots \ldots \ldots \ldots \ldots \ldots \ldots \ldots \ldots \\
12.5 \text { to } 25 \ldots \ldots \ldots \ldots \ldots \ldots \ldots\end{array}$ & $\begin{array}{c}\text { Parts in } 10^{9} \\
+70.8 \\
+18.2 \\
\quad+5.0 \\
\quad+2.0\end{array}$ & $\begin{array}{c}\text { Parts in } 10^{9} \\
+5.7 \\
+14.9 \\
+4.3 \\
\quad+1.2\end{array}$ & $\begin{array}{l}\text { Parts in } 10^{9} \\
\quad+84.5 \\
+23.2 \\
\quad+6.7 \\
\quad+1.9\end{array}$ \\
\hline Change in voltage & $\gamma_{1000 \mathrm{~A}}^{\prime \prime}$ & $\gamma_{1000 \mathrm{~B}}^{\prime \prime}$ & $\gamma_{1000 \mathrm{C}}^{\prime \prime}$ \\
\hline 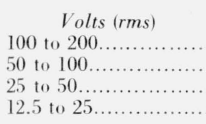 & $\begin{array}{c}\text { Parts in } 10^{9} \\
+0.1 \\
.0 \\
+.3 \\
+.6\end{array}$ & $\begin{array}{c}\text { Parts in } 10^{9} \\
\quad+1.2 \\
\quad+1.1 \\
\quad+1.1 \\
\quad+0.3\end{array}$ & $\begin{array}{c}\text { Parts in } 10^{9} \\
\quad+2.3 \\
\quad+2.2 \\
\quad+1.6 \\
\quad+0.8\end{array}$ \\
\hline
\end{tabular}

TABLE 4. Instabilities in the voltage dependence of capacitors $100 \mathrm{~A}, 100 \mathrm{~B}$, and $100 \mathrm{C}$

All values correspond to a change in voltage from 100 to $200 \mathrm{~V}$. rms. Standard deviation $=2 \times 10^{-10}$. Systematic error $<2 \times 10^{-10}$.

\begin{tabular}{c|c|c|c}
\hline \hline Time & $\gamma_{100 \mathrm{~A}}^{\prime}-\gamma_{100 \mathrm{~B}}^{\prime}$ & $\gamma_{100 \mathrm{~B}}^{\prime}-\gamma_{100 \mathrm{C}}^{\prime}$ & $\gamma_{100 \mathrm{C}}^{\prime}-\gamma_{100 \mathrm{~A}}^{\prime}$ \\
\hline Months & Parts in $10^{9}$ & Parts in $10^{9}$ & Parts in $10^{9}$ \\
0 & -0.5 & +0.9 & -0.4 \\
1 & -.6 & +.8 & -.2 \\
8 & -6.1 & +7.1 & -1.0 \\
\hline Time & $\gamma_{100 \mathrm{~A}}^{\prime \prime}-\gamma_{100 \mathrm{~B}}^{\prime \prime}$ & $\gamma_{100 \mathrm{~B}}^{\prime \prime}-\gamma_{100 \mathrm{C}}^{\prime \prime}$ & $\gamma_{100 \mathrm{C}}^{\prime \prime}-\gamma_{100 \mathrm{~A}}^{\prime \prime}$ \\
\hline Months & Parts in $10^{9}$ & Parts in $10^{9}$ & Parts in $10^{9}$ \\
0 & -0.2 & +0.4 & -0.2 \\
1 & +.1 & -.0 & -.1 \\
8 & +.5 & -.4 & -.1 \\
\hline
\end{tabular}

The values of $\gamma^{\prime}$ in tables 2 and 3 can be used to determine the functions which relate capacitance to voltage. If we assume the function

$$
C_{V}=C_{0}\left(1+k V^{m}\right)
$$

where $C_{V}$ is the capacitance at voltage $V$ and $C_{0}$ is the capacitance at zero voltage, then the values of $\gamma^{\prime}$ in each column of tables 2 and 3 will be in the ratio of $2^{3 m}: 2^{2 m}: 2^{m}: 1$. Values of $m$ were calculated from the measured values, $\gamma_{100 \mathrm{~A}}, \gamma_{100 \mathrm{~B}}, \gamma_{1000_{\mathrm{A}}}$, etc., and also from the measured differences, $\gamma_{100_{\mathrm{A}}}-\gamma_{100 \mathrm{~B}}, \gamma_{1000_{\mathrm{A}}}$ $-\gamma_{1000 \mathrm{~B}}$, etc., which had smaller uncertainties of essentially different origin (see sec. 4.1). The results of the two types of calculations differed only slightly. The calculations yielded $m \approx 3 / 2$ for capacitors $100 \mathrm{~A}$, $100 \mathrm{~B}$, and $100 \mathrm{C}$ and $m \approx 2$ for capacitors $1000 \mathrm{~A}$, $1000 \mathrm{~B}$, and 1000C.

As a partial check on the overall measuring system, the value of $\bar{\gamma}_{1000} \equiv\left(\gamma_{1000_{\mathrm{A}}}+\gamma_{1000 \mathrm{~B}}+\gamma_{1000 \mathrm{C}}\right) / 3$ from 100 to $200 \mathrm{~V}$ was determined by two methods: (1) Indirectly by measuring the difference, $\bar{\gamma}_{1000}-\bar{\gamma}_{100}$, as described in section 4.2 , (2) directly by using capacitors $1000 \mathrm{~A}, 1000 \mathrm{~B}$, and $1000 \mathrm{C}$ in bridge 3 of the three-bridge-circuit (sec. 3). The results differed by less than $3 \times 10^{-10}$.
Capacitors were chosen from more than two dozen capacitors on the basis of stability ascertained by difference measurements, similar to those described in section 4.1. The voltage dependence of capacitors $100 \mathrm{~A}, 100 \mathrm{~B}, 100 \mathrm{C}, 1000 \mathrm{~A}, 1000 \mathrm{~B}$, and 1000C was found to be considerably more stable than that of the other capacitors. The measurements were limited to $100 \mathrm{pF}$ capacitors and $1000 \mathrm{pF}$ capacitors. The use of the drifter circuit (sec. 4.1) eliminated the need for special temperature control of the capacitors.

The earliest measurements involved three commercial $100 \mathrm{pF}$ air capacitors of parallel plate design. The values obtained from difference measurements were observed to depend not only on time but also on the voltages which had previously been applied to the capacitors. In addition, a sudden, nonreversible change in capacitance of $14 \mathrm{ppm}$ was observed in one of the capacitors when the applied voltage was increased beyond $50 \mathrm{~V}$ rms. The cause of the unusual behavior was found to be metallic whiskers on the tin-plated housing of the capacitor.

Difference measurements were also made on a set of ten $100 \mathrm{pF}$ capacitors of cylindrical design. The construction of these capacitors is described in reference [2]. The values obtained from the difference measurements, corresponding to a change in voltage from 80 to 160 volts, were observed to change by as much as $3 \times 10^{-8}$ over a period of a few days. Changes as large as $3 \times 10^{-8}$ were also observed when the temperature of the capacitors was increased by approximately $10{ }^{\circ} \mathrm{C}$.

A different phenomenon was observed when difference measurements were made on a set of three $100 \mathrm{pF}$ capacitors of cylindrical design which were evacuated during measurement. The time rate of change of capacitance was found to be a function of voltage. The phenomenon is believed to be related to outgassing of the epoxy which was used to insulate and support the electrodes. Measurements of the time rate of change of capacitance as a function of voltage were made as follows: (1) The bridge was balanced and the drifter circuit was adjusted so as to obtain a steady bridge balance, (2) the detector was shorted, and the voltage applied to one capacitor was reduced to zero for $30 \mathrm{sec}$, (3) the original conditions were restored and the change in bridge balance was recorded. With $160 \mathrm{~V}$ originally applied to the capacitors, the changes in bridge balance were equivalent to proportional changes in capacitance of about $1 \times 10^{-8}$.

Difference measurements, corresponding to a change in voltage from 100 to $200 \mathrm{~V}$, were made on three commercial $1000 \mathrm{pF}$ capacitors of parallel plate construction. The changes in the values obtained from the difference measurements varied from a few parts in $10^{9}$ to $2 \times 10^{-8}$ over the period of one week.

The basic design of capacitors 100A, 100B, and $100 \mathrm{C}$ is shown to approximate scale in figure 9. Critical radii differ by approximately $3.2 \mathrm{~mm}$. Line and detector electrodes are separated from the ground electrodes by four sets of three glass spacers. The force of the compressed springs is about $45 \mathrm{~N}$. 
$0 \quad 1 \quad 2 \quad 3 \quad 4 \quad 5$ CENTIMETERS

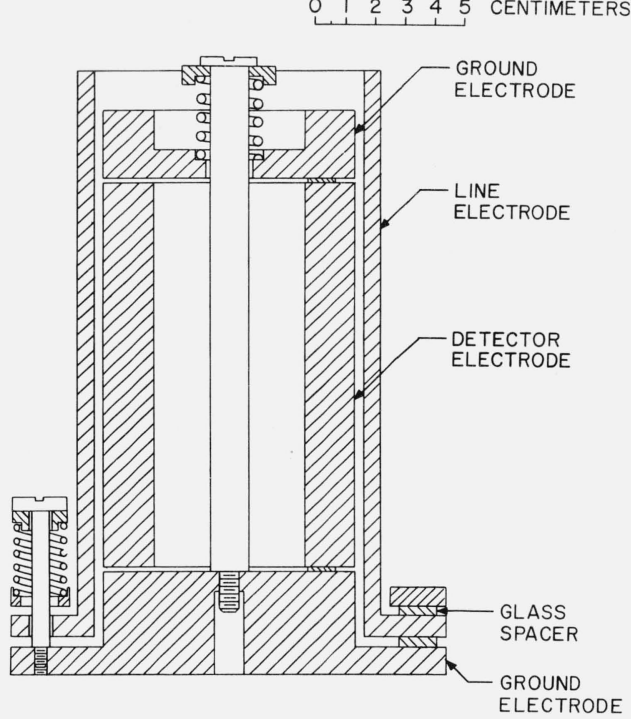

Figure 9. Cross section through the axis of capacitor 100A, 100B, or $100 C$.

It was found that after the capacitor was assembled and connected into a measuring circuit, very small radial displacements of the line electrode could be obtained by sharply tapping its base radially. This procedure was used to adjust the capacitance very close to a minimum, thus assuring that the radial components of the electrical forces were small. The design of the capacitor is such that it can easily be disassembled for cleaning or for experimental purposes. A grounded housing (not shown in the figure) is used to evacuate the capacitor.

\section{Conclusion}

The basic purpose of the present work has been to develop an accurate system for measuring both the relative and absolute voltage dependence of admittance standards. The causes of voltage dependence have been pursued only to the extent necessary to reduce instabilities. Additional measurements will be required to evaluate the instabilities which remain.
Since the mechanisms which cause instabilities in the voltage dependence of capacitors may also result in general instability, the present work has pointed out certain problem areas which should be considered in the construction of precision standards of capacitance. In addition, the present work has served as a proving ground for bridge measurements and voltage ratio measurements at the higher accuracy levels.

The author acknowledges the many suggestions of R. D. Cutkosky and the assistance rendered by D. N. Homan and Lai H. Lee in obtaining the numerical results of this paper.

\section{References}

[1] A. M. Thompson and D. G. Lampard, A new theorem in electrostatics and its application to calculable standards of capacitance, Nature 177,888(1956).

[2] M. C. McGregor, et al., New apparatus at the National Bureau of Standards for absolute capacitance measurement, IRE Trans. Instru. I-7, December 1958.

[3] N. L. Kusters and O. Petersons, The voltage coefficients of precision capacitors, IEEE Trans. Communication and Electronics $\mathbf{8 2}$ (1963).

[4] R. D. Cutkosky and J. Q. Shields, The precision measurement of transformer ratios, IRE Trans. Instr., I-9 (Sept. 1960).

[5] A. M. Thompson, The precise measurement of small capacitances, IRE Trans. Instr. I-7 (Dec. 1958).

[6] A. M. Thompson, A C bridge methods for the measurement of three-terminal admittances, IEEE Trans. Instr. and Measurement (Dec. 1964).

[7] R. D. Cutkosky, Active and passive direct-reading ratio sets for the comparison of audio-frequency admittances, J. Res. NBS 68C (Engr. and Instr.), No. 4 (Oct.-Dec. 1964). 